\title{
Cat Scratch Disease and Arthropod Vectors: More to it than a Scratch?
}

\author{
Mark Mosbacher, DVM, Sean P. Elliott, MD, Ziad Shehab, MD, Jacob L. Pinnas, MD, \\ John H. Klotz, PhD, and Stephen A. Klotz, MD
}

Purpose: Cat scratch disease is a common infection, particularly in children, and clinicians need to be aware of its potential transmission to humans by arthropod vectors such as fleas and ticks in addition to animal bites and scratches. The absence of a vertebrate bite or scratch does not preclude infection with Bartonella benselae.

Methods: Literature regarding arthropod transmission of $\boldsymbol{B}$. benselae was reviewed.

Results: B. henselae and related bacterial species are transmitted among cats and dogs by arthropod vectors. In the absence of these vectors, disease does not spread amongst the animals. On the other hand, disease can be spread to humans by bite and scratch as well as by arthropod vectors. Animals commonly infected with $\boldsymbol{B}$. henselae and arthropod vectors are discussed.

Conclusions: Clinicians should be aware that a common illness, cat scratch disease, can be transmitted by arthropod vectors and a history of an animal scratch or bite is not necessary for disease transmission. (J Am Board Fam Med 2010;23:685-686.)

Keywords: Cat Scratch Disease, Bartonella henselae, Bartonellosis, Fleas, Ticks, Bites

Several zoonotic infectious diseases remain poorly understood, their host reservoirs and means of transmission in particular. Bartonella benselae is a bacterium that has been definitively identified as the cause of cat scratch disease (CSD) within the past 20 years. Bartonella species are Gram-negative bacilli that invade endothlelial cells like Ebrlichia, Anaplasma, and Rickettsia. These latter micro-organisms are transmitted to human hosts by ticks. It is possible that Bartonella species may on occasion share with these proteobacteria the ability to be transmitted by arthropods (Figure 1). It is assumed that humans acquire $B$. benselae infection when contaminated

This article was externally peer reviewed.

Submitted 4 February 2010; revised 13 May 2010; accepted 24 May 2010.

From Third World Veterinary, Fountain Hills, AZ (MM); the Departments of Pediatrics (SPE, ZS) and Medicine (JLP, SAK), University of Arizona, Tucson; and the Department of Entomology (JHK), University of California, Riverside.

Funding: none.

Conflict of interest: none declared.

Corresponding author: Stephen A. Klotz, MD, 1501 N. Campbell Avenue, Tucson, AZ 85724 (E-mail: sklotz@u. arizona.edu). cat saliva or flea fecal material is introduced through the skin via the scratch or bite of a cat or dog. Testing for the disease among humans has centered on establishing a history of direct animal contact via exposure in an occupation or as a pet owner. A 2007 study in North Carolina of healthy immunocompetent persons with $>10$ years of reported occupational animal exposure revealed that $57 \%$ of participants had seroreactivity to 1 of 3 Bartonella antigens. ${ }^{1}$ Other studies demonstrated variable rates of Bartonella seroreactivity in $15 \%$ to $45 \%$ of healthy veterinarians, considerably higher than the $3 \%$ to $6 \%$ rates reported among the general population. Thus, direct animal contact in an occupational setting is associated with a significantly higher likelihood to develop antibodies toward $B$. henselae. However, a 2003 Austrian study did not find a statistically significant correlation between domestic pet ownership and Bartonella seroprevalance, nor were there any differences with respect to urban versus rural populations. ${ }^{2}$ Clearly there is ambiguity about CSD acquisition, and one of the as yet unproven but possible vectors may be arthropods, specifically ticks. 
The capability of a tick species (Dermacentor andersoni) to experimentally transmit $B$. bacilliformis to nonhuman primates was confirmed more than 80 years ago, when naive monkeys became infected after being bitten by ticks that had previously fed on monkeys already carrying the disease. More recent studies have identified various Bartonella species in various blood-sucking arthropods that feed on humans as atypical hosts (Table 1). A 2003 Italian study detected $B$. henselae in $1.48 \%$ of sheep ticks removed from asymptomatic persons. ${ }^{3}$ The potential for Ixodes ricinus ticks to transmit $B$. benselae in vitro was confirmed by a study in which sheep ticks were fed infected blood and transmitted the bacteria through all its developmental stages. ${ }^{4}$ However, the clinical importance of this finding has been questioned. Some authorities consider tick-borne transmission as anecdotal and not yet proven. ${ }^{5}$

Although infection of a human patient with $B$. benselae via a tick-borne vector has not yet been convincingly demonstrated, physicians may want to consider Bartonella serology for patients who present with persistent fever or lethargy and who have a history of exposure to ectoparasites (such as ticks), even in the absence of occupational exposure or pet ownership. Unless specifically screened for, $B$. henselae may remain undetected

Figure 1. Schema of present knowledge about the transmission of Bartonella benselae to man.

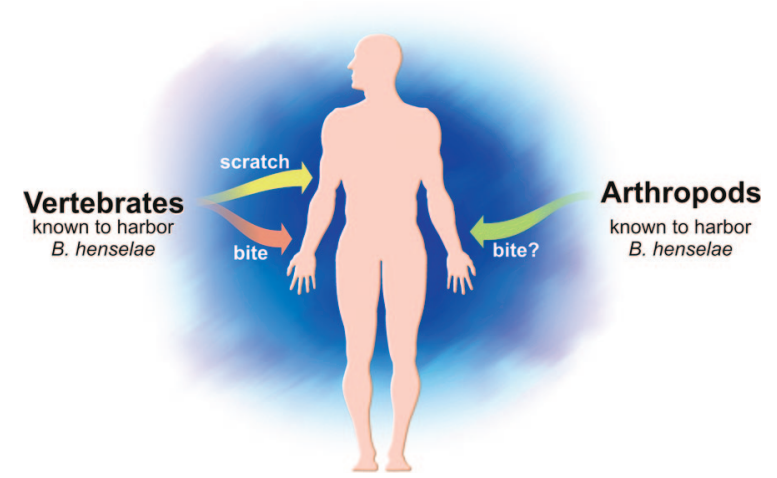

Table 1. Animal Species in Which Bartonella benselae Seroreactivity or Isolation is Reported

\begin{tabular}{ll}
\hline Vertebrates & \multicolumn{1}{c}{ Arthropods } \\
\hline Domestic cats & Cat fleas \\
Domestic dogs & Sheep ticks \\
Coyotes & Western black-legged ticks \\
Horses & Stable flies \\
Florida panthers & Deer keds \\
Beluga & \\
Porpoises & \\
\hline
\end{tabular}

in patients and CSD may remain under-diagnosed.

A picture is emerging in which Bartonella species are becoming increasingly implicated in disease with a wide variety of manifestations in humans and animal species. $B$. henselae may join the ranks of other global pathogens for which rapid human and animal travel has the potential to cause widespread disease transmission and the possibility that a sizable number of cases will go unrecognized and untreated.

\section{References}

1. Breitschwerdt EB, Maggi RG, Duncan AW, Nicholson WL, Hegarty BC, Woods CW. Bartonella species in blood of immunocompetent persons with animal and arthropod contact. Emerg Infect Dis 2007; 13:938-41.

2. Skerget M, Wenisch C, Daxboeck F, Krause R, Haberl R, Stuenzner D. Cat or dog ownership and seroprevalence of ehrlichiosis, Q fever, and catscratch disease. Emerg Infect Dis 2003;9:1337-40.

3. Sanogo YO, Zeaiter Z, Caruso G, et al. Bartonella benselae in Ixodes ricinus ticks (Acari: Ixodida) removed from humans, Belluno Province, Italy. Emerg Infect Dis 2003;9:329-32.

4. Cotté $\mathrm{V}$, Bonnet $\mathrm{S}$, Le Rhun $\mathrm{D}$, et al. Transmission of Bartonella henselae by Ixodes ricinus. Emerg Infect Dis 2008;14:1074-80.

5. Telford SR III, Wormser GP. Bartonella spp. transmission by ticks not established. Emerg Infect Dis 2010;16:379-84. 